\title{
Spatial and temporal variations in sea lice (Copepoda: Caligidae) infestations of three salmonid species farmed in net pens in southern Chile
}

\author{
Francisco J. Zagmutt-Vergara, Tim E. Carpenter*, Thomas B. Farver, Ronald P. Hedrick \\ Department of Medicine and Epidemiology, School of Veterinary Medicine, University of California, Davis, California 95616, USA
}

\begin{abstract}
Sea lice infestations have become a major health problem for farmed salmonids throughout the world including Chile. In southern Chile, 6 geographical areas, divided into 22 geographical zones with a total of 127 salmon farming centers and 1519 sea pens, were regularly sampled from December 1999 to April 2002. A linear mixed-effects model (LME) approach was used to describe the infestations of adult forms of sea lice on 3 salmonid species farmed in southern Chile. The variables fish species, water temperature, water salinity, fish weight, juvenile parasite count, pen shape, treatment status in previous month and the interaction of previous and current month treatments were found to be statistically significant fixed effects for the population sampled. The most susceptible species to sea lice infestation was rainbow trout Oncorhynchus mykiss, while the least susceptible species was coho salmon $O$. kisutch. Fishes in pens treated in the previous month with avermectins were associated with the smallest sea lice count compared to fishes in pens not treated or treated with other products. The variability in sea lice infestations in areas and zones within areas was not statistically significant when controlling for the previously mentioned fixed variables. The variability between centers, the within-pen variability, and the interaction between within-pen effect and the date of measurement were statistically significant and not explained by the fixed effects. Potential sources for this variability are discussed. We conclude that the epidemiology of sea lice infestations in farmed salmonids in southern Chile is complex and in need of further study.
\end{abstract}

KEY WORDS: Sea lice $\cdot$ Epidemiology $\cdot$ Fish health $\cdot$ Farmed salmonids $\cdot$ Linear mixed models Resale or republication not permitted without written consent of the publisher

\section{INTRODUCTION}

Salmon lice or sea lice (Lepeophtheirus spp. and Caligus spp.) are a group of marine copepod parasites that represent one of the greatest worldwide threats to salmon farming. The first cases of this parasitosis in farmed Atlantic salmon Salmo salar were reported in the 1960s in Norway (Hastein \& Bergsjo 1976), and subsequently the disease has been reported as a significant problem in almost all countries where salmon culture is present, including Scotland (Wootten 1985), Ireland (Tully 1989), Canada (Hogans 1995) and, more recently, Chile (Carvajal et al. 1998, Boxshall \& Bravo 2000).

There are few reports on the presence of sea lice in Chilean farmed salmon. The earliest report described the presence of Caligus teres on farmed coho salmon Oncorhynchus kisutch in southern Chile (Reyes \& Bravo 1983). Later, Gonzalez \& Carvajal (Gonzalez \& Carvajal 1994) described infestations on rainbow trout $O$. mykiss and coho salmon due to C. flexispina, a species that had previously been reported in Chile only from native fish species of Easter Island (Fernandez \& Villalba 1986). Carvajal et al. (1998) reported that C. flexispina was the dominant caligid species on salmonids farmed in southern Chile, and especially among rainbow trout. Gonzales et al. (2000) also found C. flexispina as the major copepod species in Chilean salmon and showed that the duration of its life cycle depends on the fish-host species. Recently it was reported that the dominant caligid species, previously 
named $C$. flexispina, was in fact a new species, $C$. rogercresseyi (Boxshall \& Bravo 2000). No studies have described the effects of different environmental and management factors that may influence sea lice burdens on salmonids cultured in southern Chile. Univariate analyses of such environmental and management factors are common in the literature for salmonids cultured in northern hemisphere countries (Jaworski \& Holm 1992, Hogans 1995, Boxaspen 1997, Jackson et al. 1997, Rivie et al. 2002). However, these reports ignore the nested structure of the data sets from salmon farms, i.e. pens within farms and farms within geographical regions. Furthermore, data sets reporting sea lice burdens are generally based on random samples at different levels (i.e. random sample of fish from a particular pen and random samples of pens within a farm) that allow the researcher to extrapolate the results to the population from which the samples were obtained.

Some univariate statistical analyses do not discriminate between random effects and fixed effects, leading to incorrect generalizations of the data. The results from these analyses in aggregated parasite distributions are much more likely to produce Type I errors and, to a lesser degree, Type II errors (Wilson et al. 1996). A method that takes into account the nested nature of the sea lice infestation on farmed salmonids is multilevel analysis, such as linear mixed-effects model (LME). This approach allows the researcher to include both fixed effects, which are parameters associated with an entire population (e.g. school, herd or pen) or with certain repeatable levels of experimental factors, and random effects, which are parameters that are obtained from random samples drawn out of a population and therefore cannot be reproduced by the researcher (Pinheiro \& Bates 2000). Mixed models are particularly appropriate for epidemiologic studies because their flexible covariance structure allows nonconstant correlation among the observations and/or unbalanced data (Lindstrom \& Bates 1990).

The objective of our study was to assess differences in the mean count of adult forms of sea lice as found on 3 species of farmed salmonids in southern Chile. The salmon were sampled over a 29 mo period for the presence of sea lice, along with data on several management and environmental parameters and other spatial and temporal factors.

\section{MATERIALS AND METHODS}

Location and database. The data used in this study were obtained from the electronic records of the 'Chilean Monitoring Program for Sea Lice' managed by the Instituto Tecnológico del Salmón (Intesal SA), from December 1999 to April 2002 inclusive. The data set consisted of 14356 records based on samples drawn every 2 wk from sea pens containing the 3 main species of salmonids farmed in southern Chile, Atlantic salmon Salmo salar, rainbow trout Oncorhynchus mykiss and coho salmon $O$. kisutch. Sampling was conducted in 6 main geographical areas (Fig. 1), each of which was divided into geographical zones (1 to 8 zones per area, 22 zones in total) involving 127 salmon farming centers with a total of 1519 pens sampled. From each center a random sample of 10 to 30 fishes was drawn, using a hand net, from each of 4 to 10 pens. Fishes were anesthetized in a container using a solution of 2-phenoxy-ethanol, and the total numbers of adult and juvenile forms of sea lice attached were counted for each fish (juvenile defined as any developmental stage previous to adult stage). Detached lice were counted in the anesthesia container. Weight was recorded for each fish sampled. The data recorded from these samples were the mean count of adult lice attached fish ${ }^{-1}$ pen $^{-1}$, the mean count of juvenile lice attached fish ${ }^{-1}$ pen $^{-1}$ and the mean weight $(\mathrm{kg})$ of fish ${ }^{-1}$

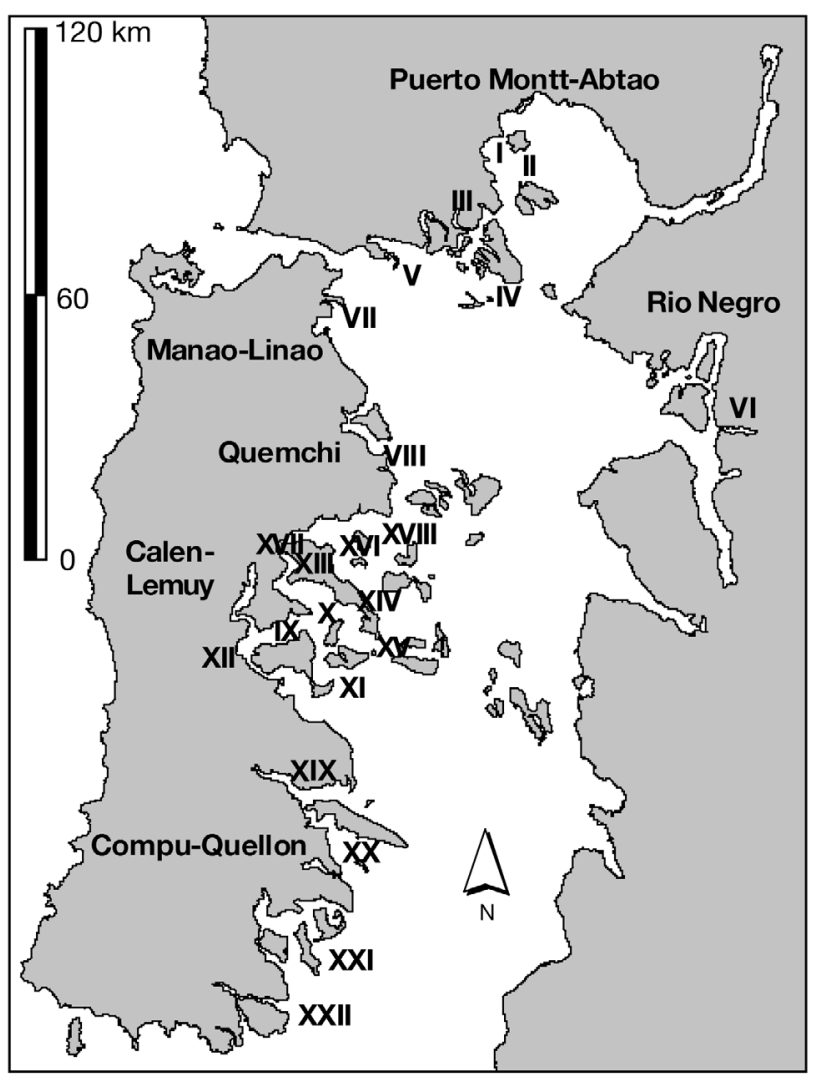

Fig. 1. Areas and zones included in Chilean sea lice monitoring program. Mean counts of sea lice adults and juveniles made every 15 d from December 1999 to April 2002 in 1519 sea pens in southern Chile in 6 geographical areas and 22 geographical zones (roman numerals) 
pen $^{-1}$. Additional data recorded included sample date, pen shape (round or square), location (extreme or center of the group of pens), pen diameter or width (m), previous harvest status of the pen (i.e. harvested or not), fish species held in each pen, treatments applied against sea lice, and the product used. Also, sequential registers of water temperature $\left({ }^{\circ} \mathrm{C}\right)$ and salinity in parts per thousand (ppt) measured at $5 \mathrm{~m}$ depth, obtained from a monitoring program of environmental parameters in parallel to the sea lice program, were available for about $20 \%$ of the farming centers.

Statistical analysis and data management. The original data set was compiled and arranged using Excel 2002 and Access 2002 (both from Microsoft). All modifications made to the data set were double-checked and compared to the original registers. Observations with missing values in any category were deleted. Pivot tables were used to check double entries. Each pen received a unique identification number and every time a pen was harvested and repopulated it was recoded as a new pen. Any pen with less than 3 registers was excluded from the analysis to avoid inaccuracies in the random parameters estimates. Variables 'temperature' and 'salinity' were created joining the original database to the environmental data set. For categorizing the temporal application of treatments for sea lice, 4 new variables were created 'treatment status in current time period', 'treatment status 1 mo earlier', 'treatment status 2 mo earlier' and 'treatment status 3 mo earlier' (Table 1). The treatments were assumed to be administered to the pen at the midpoint of the relevant $2 \mathrm{wk}$ period.

Exploratory analyses including histograms for each variable, and basic descriptive statistics were performed to assess the normality of the continuous variables and to find any incorrect data entry. The distribution of the mean adult parasite count was analyzed based on an $\mathrm{ln} \cdot$ (adult mean + 0.1) transformation to provide a better approximation to normality. Any observation falling beyond 4 standard deviations from the mean was assumed to be a data entry error and hence was deleted.

Linear mixed-effects models. The models were fitted using the nlme3 package from the statistical software

S-Plus, Version 6.03, Release 2 (Insightful), using restricted maximum-likelihood estimations of model parameters. All the following statements in this section referring to adult count represent $l n \cdot$ (mean adult +0.1 ) . We started modeling the adult count $y_{k l}$ measured on the 1 th pen within the $k$ th center as being linearly dependent on several fixed and random effects, obtaining the model:

$$
y_{k l}=\beta_{0}+\beta_{n}+b_{k}+\varepsilon_{l(k)}
$$

where $\beta_{0}$ is the intercept, $\beta_{n}$ is the vector of the $n$ fixed effects (Table 1$), b_{k}$ is the vector of the random effects for Center $k$ and $\varepsilon_{l(k)}$ is the vector of the random effects for Pen $l$ within Center $k$ or the within-group errors.

The model assumes that there is independence between the random effects and the errors at each level, there is equal variance among the errors at each

Table 1. Variables and their respective levels included in the initial linear mixed model used to evaluate sea lice burdens on farmed salmonids in southern Chile measured every 15 d from December 1999 to April 2002

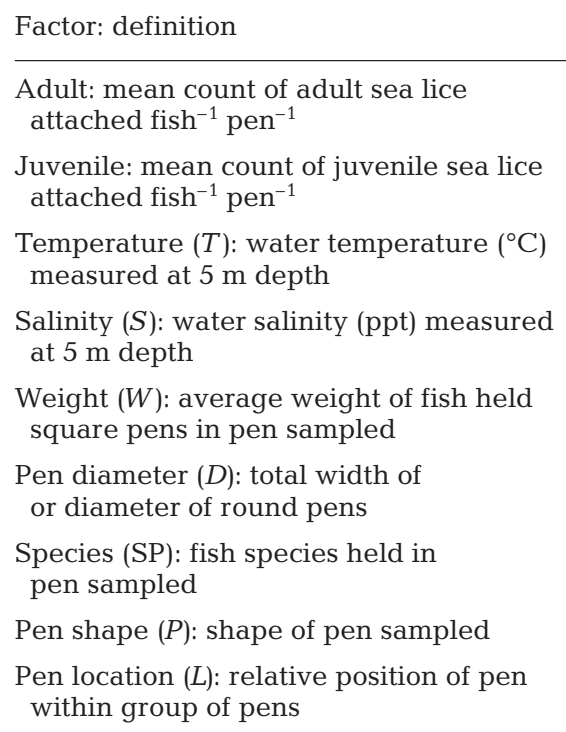

Juvenile: mean count of juvenile sea lice attached fish ${ }^{-1}$ pen $^{-1}$

Temperature $(T)$ : water temperature $\left({ }^{\circ} \mathrm{C}\right)$ measured at $5 \mathrm{~m}$ depth

Salinity $(S)$ : water salinity (ppt) measured at $5 \mathrm{~m}$ depth

Weight $(W)$ : average weight of fish held square pens in pen sampled

Pen diameter $(D)$ : total width of or diameter of round pens

Species (SP): fish species held in pen sampled

Pen shape $(P)$ : shape of pen sampled

Pen location $(L)$ : relative position of pen within group of pens

Harvested $(H)$ : pen previously harvested

Current treatment (TR): treatment against sea lice at midpoint of current 2 wk period

Treatment status 1 mo earlier (TR.1): treatment status at midpoint of period 1 mo prior to current treatment

Treatment status 2 mo earlier (TR.2): treatment status at midpoint of period 2 mo prior to current treatment

Treatment status 3 mo ealier (TR.3): treatment status at midpoint of period 3 mo prior to current treatment
$5 \mathrm{~m}, 12 \mathrm{~m}, 15 \mathrm{~m}, 16 \mathrm{~m}$, $20 \mathrm{~m}, 30 \mathrm{~m}$

Atlantic salmon; rainbow trout; coho salmon

Round or square

Extreme: pen located at 1 of 2 extremes of group of pens;

Center: pen not located at an extreme

Yes/No

No treatment

Avermectins

Other: treatment with product other than avermectins

Same as current treatment

Same as current treatment

Same as current treatment 
group (homoscedasticity) and that random effects and errors follow an approximately normal distribution having mean zero and variances, $\sigma_{i}^{2}$. This is expressed as $b_{k} \sim N\left(0, \sigma^{2}\right)$ and $\varepsilon_{l(k)} \sim N\left(0, \sigma^{2}\right)$.

In practical terms, the fixed effects represent the contribution of each variable included in the model throughout the population of pens sampled and the random effects represent the contribution to the variability of the adult count at each level of nesting. Therefore, the between-centers effect $b_{k}$ is a random variable representing the deviation of the mean adult count per center from the mean adult count of the population of centers in southern Chile from which the centers were sampled, and the within-pen effect $\varepsilon_{l(k)}$ is a random variable representing the deviation of the average adult count for the 1 th pen, from the mean average adult count of the $k$ th center.

In general, a positive value for the random effects estimates indicates variability in each level of the hierarchy. Hence, the estimates obtained for the random effect $b_{k}$ represent the variability between centers not explained by the fixed effects included in the model, and the estimates obtained for the error term $\varepsilon_{l(k)}$ represent the variability among pens within Center $k$ not explained by the variables included in the model.

From the initial model, F-tests were performed to assess the overall significance of each fixed effect. Terms were deleted using a backward elimination procedure constrained by the hierarchical principle (Kleinbaum 1994). Thus non-significant ( $p>0.05$ ) 3-way interaction terms were first considered for removal, then 2-way interaction terms and, lastly, main effects. When there were multiple non-significant terms for removal at a step, the term removed was that with the largest p-value. Variables with similar nonsignificant levels were deleted together. After each deletion the model was refitted. The final model was obtained when all the variables included in the model were statistically significant $(p<0.05)$. If a variable were borderline non-significant but the relationship was considered to be biologically important, the term was maintained in the model. Potential confounders were tested against the final model to reveal meaningful differences in the parameter estimates after including the confounders in the model. If the estimate varied by more than $20 \%$, the factor was kept in the model to adjust for confounding. Contrasts were used for the categorical covariates to test the differences of each level of the factor compared to a baseline level, e.g. current treatment 'no treatment' versus 'avermectins' and 'other' versus 'avermectins'. The statistical significance of the contrast comparisons was tested using approximate Student's $t$-tests. Differences between 2 levels of a factor that were not compared using the contrasts parameterization, e.g. current treatment 'avermectins' versus 'other', were evaluated using approximated F-tests. Random effects were tested to determine their individual contribution to the goodness-of-fit of the model. First, as the literature describes an important seasonality in the sea lice counts, we tested the interaction between the fixed effect 'date' and the random effect 'pen' generating a repeated-measures model. Thus, we included an extra parameter to the random effects formula, obtaining:

$$
y_{k l m}=\beta_{0}+\beta_{n}+b_{k}+b_{l(k)}+\varepsilon_{m(k l)}
$$

that is a new parameterization of Eq. (1) with an extra term $m$, which represents the additional level of nesting for the date $m$ within Pen 1 . Then we tested this model against Model 1 using a likelihood ratio test based on the Akaike information criterion (AIC) and the Bayesian information criterion (BIC). If the difference in both criteria between the models were not significant, we kept the simpler model (the model with fewer parameters). The same procedure was used to test the need to include extra random effects in the model. Therefore, in addition to the simpler model with pens nested within centers, we created a model including 2 extra hierarchy levels, 'geographical areas' and 'zones' within geographical areas.

Hence, this model was:

$$
y_{i j k l m}=\beta_{0}+\beta_{n}+b_{i}+b_{j(i)}+b_{k(i j)}+b_{l(i j k)}+\varepsilon_{m(i j k l)}
$$

Eq. (3) is an extension of Eq. (2) with 2 extra parameters, $i$ and $j$, that represent the additional random effects levels of nesting for the $j$ th zone within the $k$ th geographical area.

Approximate $95 \%$ confidence intervals (CI) were computed to check the precision of the final model's random-effects estimates. The overall goodness of fit of the model was assessed using diagnostics plots of response versus fitted values. Quantile-quantile (q-q) plots of the residuals versus normal distribution were examined to check the assumptions of normality at each level of the random effects. In addition, a histogram of the residual was used to check the assumption of the normality of the residuals at the within-pen level.

\section{RESULTS}

\section{Data summary}

A total of 2005 complete records was obtained from the original 14356 records. The main reason for deletions was missing values on 'temperature' and 'salinity' (10 648 missing values), and combined missing values for 'Pen shape', 'Harvested', and 'Placing' (1668 missing values). Only 35 pens were deleted due to less than 
Table 2. Summary of count of adult sea lice in farmed salmonids in southern Chile measured every $15 \mathrm{~d}$ from December 1999 to April 2002 in geographical areas and zones included in the analyses

\begin{tabular}{|lccc|}
\hline Area & Zone & Mean & (SD) \\
\hline Calen - Lemuy & Area mean & $\mathbf{2 . 1 9}$ & $\mathbf{( 3 . 8 3 )}$ \\
& IX & 2.43 & $(5.02)$ \\
& X & 1.47 & $(2.44)$ \\
& XII & 0.75 & $(0.93)$ \\
& XVI & 2.66 & $(4.33)$ \\
Xuerto Montt-Abtao & Area mean & $\mathbf{3 . 5 4}$ & $\mathbf{( 5 . 8 4 )}$ \\
& II & 6.00 & $(11.01)$ \\
& IV & 3.69 & $(5.03)$ \\
Quemchi & V & 2.70 & $(4.50)$ \\
& Area mean & $\mathbf{4 . 4 6}$ & $\mathbf{( 8 . 9 1 )}$ \\
Over all areas & VIII & 4.46 & $(8.91)$ \\
& Area mean & $\mathbf{3 . 3 8}$ & $\mathbf{( 6 . 3 6 )}$ \\
\hline
\end{tabular}

3 registers in the database. Table 2 shows the mean count of adult sea lice for the geographical areas and zones within areas finally used in the analyses. The mean counts did not differ substantially between areas and varied more between zones within areas. The mean adult count over all areas was 3.38, with a standard deviation (SD) of 6.36. In all the cases the standard deviation was larger than the mean, indicating that mean adult counts were overdispersed.

Fig. 2 shows the distribution of the log-transformed mean adult count among the centers included in the analyses. There was considerable variability in mean adult counts between the centers sampled. The 25 and 75 percentiles were very broad and the median value was not constant among the centers. The smallest median value of the log-transformed parasite count was approximately -2.3 (this is close to 0 adults fish ${ }^{-1}$ ) and the largest was approximately 2.8 (close to 16.3 adults fish $^{-1}$ ). Centers 35, 24 and 20 reported small counts that were consistent throughout the sampling period, and therefore the quartiles for these centers were very narrow.

Fig. 3 shows the smoothed mean count for adult and juvenile sea lice over all the pens sampled at each date. Adult and juvenile counts were highly correlated ( $\mathrm{r}=$ 0.59 ) over the study period. The highest peak occurred at the end of March in the Year 2000, with a mean count close to 12 parasites for both developmental stages. In the following periods the mean lice count systematically decreased until October 2000, reaching a mean level of approximately 1 parasite fish ${ }^{-1}$. The mean lice count increased again throughout the spring and summer 2000-2001, reaching a peak of 4.4 mean parasites; the lice counts generally decreased from March 2001 and April 2001 for the adult and juvenile parasites respectively, to the end of the recording.

\section{Fitted model}

The final model is summarized in Table 3 for the overall contribution of the fixed effects and Table 4 for the random effects. The main effects 'pen diameter', 'pen location', 'harvested', 'treatment 2 mo earlier' and 'treatment 3 mo earlier' were statistically non-significant $(p>0.05)$ and were deleted from the model. After the deletions the only interaction term kept in the model was 'current treatment' $x$ 'treatment 1 mo earlier' ( $p<0.0001)$. Although the main effect 'current treatment' was non-significant ( $p=0.123)$, it was retained in the model because of the significant interaction involving this term, which is consistent with the

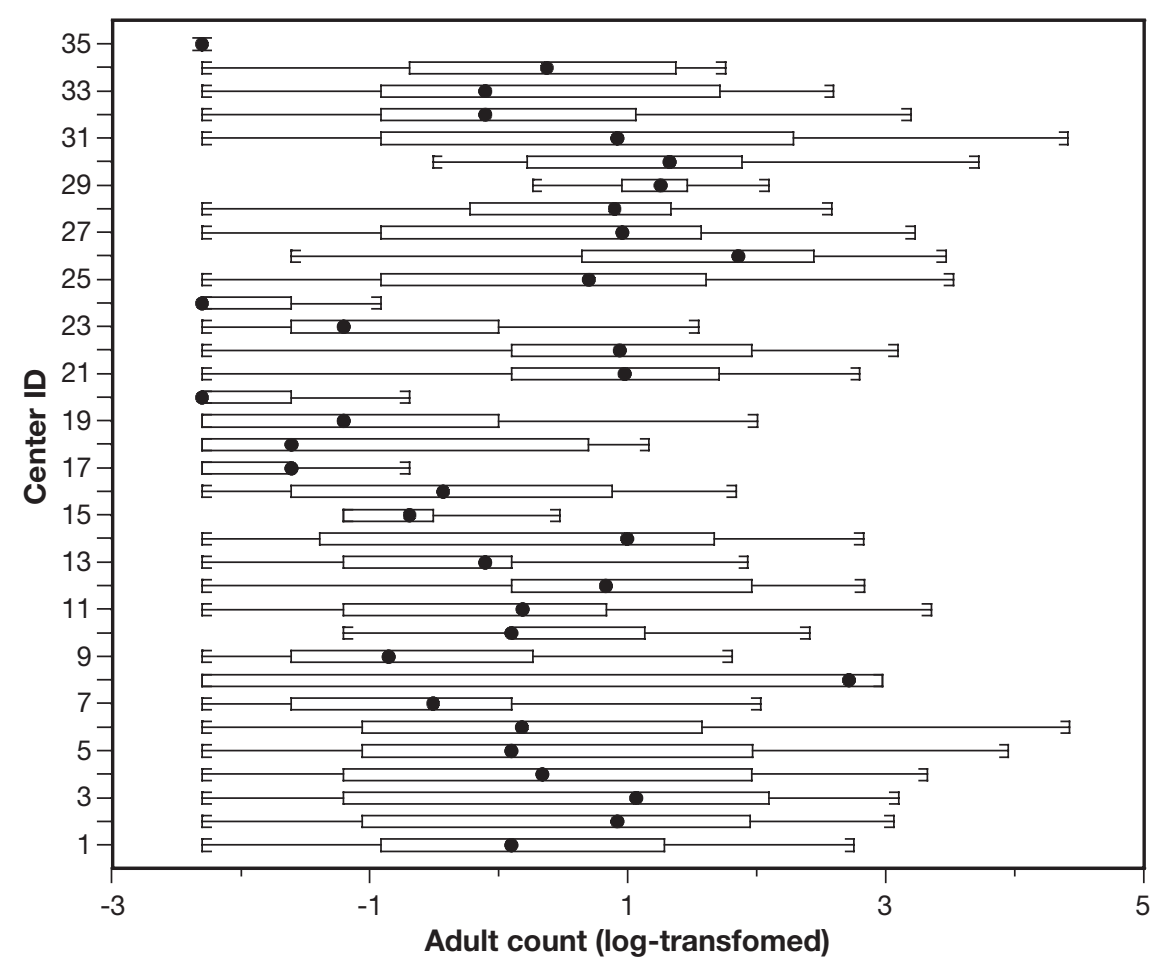

Fig. 2. Box-plot of log-transformed mean (+0.1) count of adult sea lice fish $^{-1}$ measured every 15 d from December 1999 to April 2002, in total of 35 salmonid farming centers. (•) Median (50th percentile); 25th and 75th percentiles; whiskers: maximum and minimum values; ID: identification number 


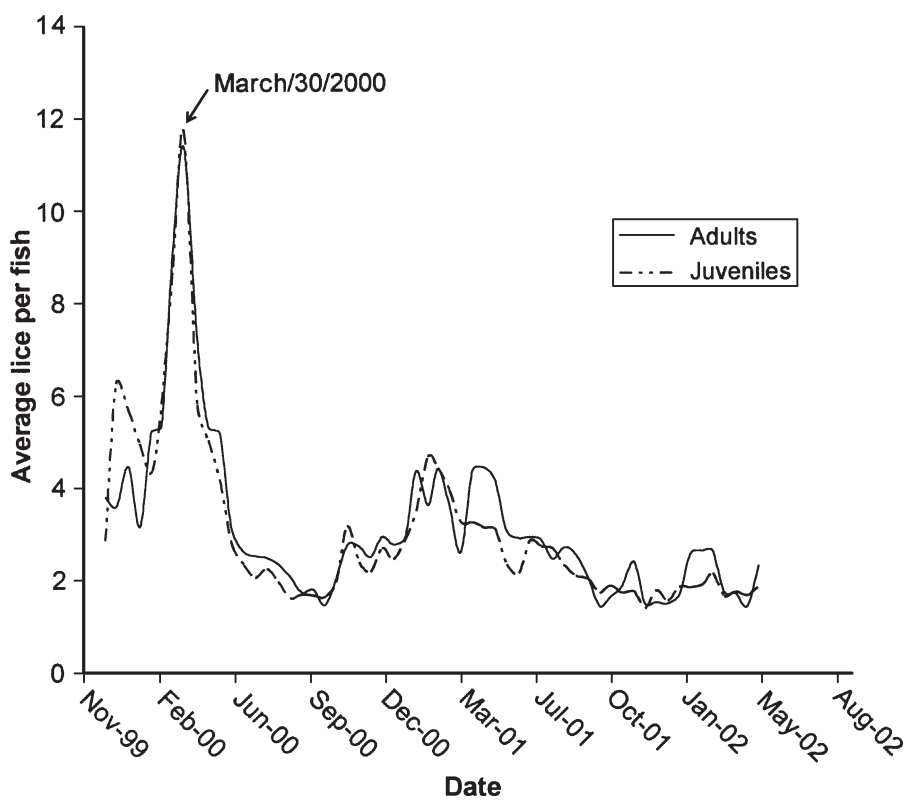

Fig. 3. Smoothed mean sea lice count per fish measured every 15 d from December 1999 to April 2002 in 1519 sea pens

hierarchical principle (Kleinbaum 1994). None of the quadratic terms was statistically significant ( $p>0.05)$. The model including a random interaction term of date and pen (Model 2) had an AIC score of 6523 and a BIC score of 6646 . The model without this interaction term had AIC and BIC scores of 6543 and 6660, respectively. The likelihood-ratio test results for these comparisons were highly significant $(p<0.001)$, suggesting that retention of the interaction term significantly improved the fit of the model. Model 3, which included the hierarchy levels 'geographical areas' and 'zones' within geographical areas as random effects, was compared to the reduced model (Model 2). Model 3 had an AIC score of 6523 and a BIC score of 6661. The likelihoodratio test for the comparison of Models 2 and 3 was not significant $(p>0.999)$ and therefore the simpler Model 2 was chosen.

Table 3. Fixed effects included in final model used to evaluate sea lice burdens on farmed salmonids in southern Chile

\begin{tabular}{|c|c|c|c|c|}
\hline Source & No. & $\begin{array}{l}\text { df } \\
\text { Density }\end{array}$ & $F$-value & $\mathrm{p}$ \\
\hline Fish species & 2 & 345 & 73.704 & $<0.0001$ \\
\hline Temperature & 1 & 345 & 22.746 & $<0.0001$ \\
\hline Salinity & 1 & 345 & 3.895 & 0.0492 \\
\hline Fish weight & 1 & 345 & 135.288 & $<0.0001$ \\
\hline Juvenile parasite count & 1 & 345 & 491.025 & $<0.0001$ \\
\hline Current sea lice treatment & 2 & 345 & 2.107 & 0.1232 \\
\hline Treatment status 1 mo earlier & 2 & 345 & 21.481 & 0.0001 \\
\hline Pen shape & 1 & 345 & 7.696 & 0.0058 \\
\hline \multicolumn{5}{|l|}{ Current treatment $x$} \\
\hline Treatment status 1 mo earlier & 4 & 345 & 5.390 & 0.0003 \\
\hline
\end{tabular}

Table 4. Random effects included in final model used to evaluate sea lice burdens on farmed salmonids in southern Chile

\begin{tabular}{|lccc|}
\hline Random effect & N & SD & $95 \%$ CI \\
\hline Between-centers $\left(b_{k}\right)$ & 35 & 0.462 & $(0.340,0.626)$ \\
Between-pens $\left(b_{l(k)}\right)$ & 1643 & 0.004 & $(0.001,0.232)$ \\
Residual or within-pens $\left(\varepsilon_{m(k l)}\right)$ & 2005 & 1.181 & $(1.145,1.219)$ \\
\hline
\end{tabular}

Table 4 shows that the variability in log-transformed mean count of adult sea lice (expressed as SD) between centers (not explained by the fixed covariates in the final model, see Table 3) was 0.462 . The SD of the response between pens nested within a center (not explained by the fixed covariates) was 0.004 . The estimated SD of the count of adult sea lice within pens nested within a center (not explained by the fixed covariates) was 1.181, the largest for the random effects. The results for the random effects agree with the variability observed in the box plots for the salmon farming centers (Fig. 2), the pens within centers and in the summary data for each hierarchy (results not shown due to the large number of centers and pens). None of the $95 \%$ CIs for the estimates included zero, meaning that in all the hierarchy levels there is significant variability not taken into account by the covariates included in the model.

Table 5 gives the estimates of the slope parameters for the fixed effects in the model. The interpretation of these parameters in the case of the continuous variables, using 'temperature' as an example, is that for every increase of $1^{\circ} \mathrm{C}$ in the water temperature the model estimates a significant $(p=0.002)$ increase in the log-transformed mean adult parasite count of almost 0.065 , other variables held constant. The mean juvenile sea lice count, the average fish weight, and water salinity, the other continuous variables in the model, also exhibited positive relationships with mean adult parasite count. The relationship of the mean log-transformed count of adult sea lice with average fish weight and mean juvenile sea lice count were highly significant $(\mathrm{p}<0.0001)$.

Using species as an example of a categorical variable, Table 5 shows that with all other variables held constant, the mean logtransformed adult parasite count in Atlantic salmon was 1.163 parasites higher than for coho salmon while the mean log-transformed adult parasite count in rainbow trout was 2.267 parasites higher than for coho salmon. Both of these comparisons were highly significant $(\mathrm{p}<0.0001)$.

The interpretation of the other categorical variables in the final model ('current treatment status' and 'treatment status 1 mo ear- 
Table 5. Fixed-effects estimates using comparisons with a baseline to evaluate sea lice burdens on farmed salmonids in southern Chile. Estimates of slope $\left(\beta_{i}\right)$ are log of adult count +0.1 , where baseline is second factor level in comparisons (in parentheses); TR: current treatment; TR.1: treatment status 1 mo earlier

\begin{tabular}{|lrrr|}
\hline Comparison & $\hat{\beta}_{i}(\mathrm{SE})$ & $t$-value & $\mathrm{p}$ \\
\hline Atlantic vs. coho & $1.163(0.284)$ & 4.1 & $<0.0001$ \\
Trout vs. coho & $2.267(0.288)$ & 7.9 & $<0.0001$ \\
Temperature $\left({ }^{\circ} \mathrm{C}\right)$ & $0.065(0.021)$ & 3.1 & 0.0020 \\
Salinity $(\mathrm{ppt})$ & $0.044(0.026)$ & 1.7 & 0.0894 \\
Weight $(k)$ & $0.224(0.021)$ & 10.8 & $<0.0001$ \\
Juveniles & $0.146(0.007)$ & 20.9 & $<0.0001$ \\
TR no treatment vs. avermectins & $-1.490(0.305)$ & -4.9 & $<0.0001$ \\
TR other vs. avermectins & $-1.575(0.542)$ & -2.9 & 0.0039 \\
TR.1 no treatment vs. avermectins & $-0.796(0.305)$ & -2.6 & 0.0093 \\
TR.1 other vs. avermectins & $-0.434(0.405)$ & -1.1 & 0.2847 \\
Pen-shape square vs. round & $-0.295(0.274)$ & -1.1 & 0.2834 \\
TR no treatment TR.1 no treatment vs. & & & \\
TR avermectins TR.1 avermectins & $1.458(0.321)$ & 4.5 & $<0.0001$ \\
TR other TR.1 no treatment vs. & $1.541(0.558)$ & 2.8 & 0.0061 \\
$\quad$ TR avermectins TR.1 avermectins & & & \\
TR no treatment TR.1 other vs. & $1.406(0.429)$ & 3.3 & 0.0012 \\
$\quad$ TR avermectins TR.1 avermectins & & & \\
TR other TR.1 other vs. & $1.268(0.646)$ & 1.9 & 0.0506 \\
TR avermectins TR.1 avermectins & & & \\
\hline
\end{tabular}

lier') is more complex due to the significant interaction between them. The meaning of the interaction itself is confounded due to the uncertainty present in the category 'others'. Because of this uncertainty, the general interpretation of all the comparisons used for this interaction term is that the mean log-transformed adult parasite count for pens currently treated with avermectins and also treated 1 mo earlier with avermectins had a significantly $(\mathrm{p}<0.05)$ smaller mean count of adult sea lice than any combination of pens (either not being currently treated or treated with other products), that 1 mo earlier were either not treated or were treated.

The results of the F-tests used to evaluate the differences between 2 estimates that were not compared using contrasts were significant for the comparison of species 'rainbow trout' versus 'Atlantic salmon' $(p<0.05)$. None of the other comparisons were statistically significant $(\mathrm{p}>0.05)$.

\section{Goodness-of-fit of final model}

Fig. 4 showed that the model exhibited good fit. The straight, diagonal line demonstrates that every predicted response from the model was very close to the actual observed response value. The normal probability plot of the residuals (Fig. 5) showed that the residu- als were approximately normally distributed. The q-q plots for each random level did not show any departure from the normality assumption (results not shown).

\section{DISCUSSION}

All the following conclusions and discussions for the random effects are generalized to the population of farmed salmonids in southern Chile in the locations from which samples were obtained for this study. Extension beyond this population may or may not be valid.

\section{Random effects}

Variability between geographical areas and zones

Significant geographic variability has been reported in the infestations of sea lice among cultured salmon populations of several countries (Jaworski \& Holm 1992, Hogans 1995, Boxaspen 1997, Jackson et al. 1997). However, none of these studies adjusted their results by the effect of different management and environmental factors, and hence the spatial effect reported might have been confounded by such factors (i.e. the variability observed between geographical areas might be due to the different water temperatures and not to hypothesized differences in management between the areas). The statistically non-significant

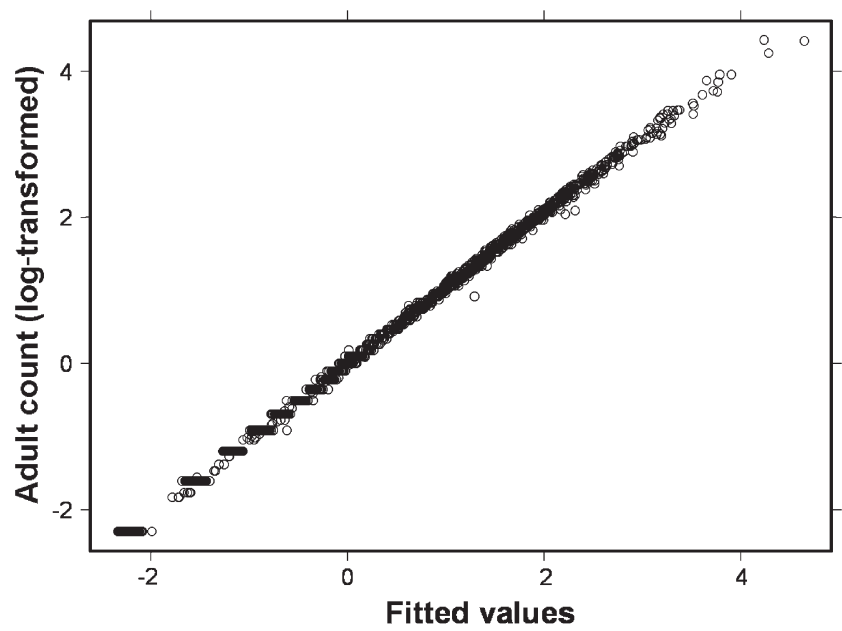

Fig. 4. Response (log-transformed mean adult count) versus fitted values from final model used to evaluate sea lice burdens on farmed salmonids in southern Chile 


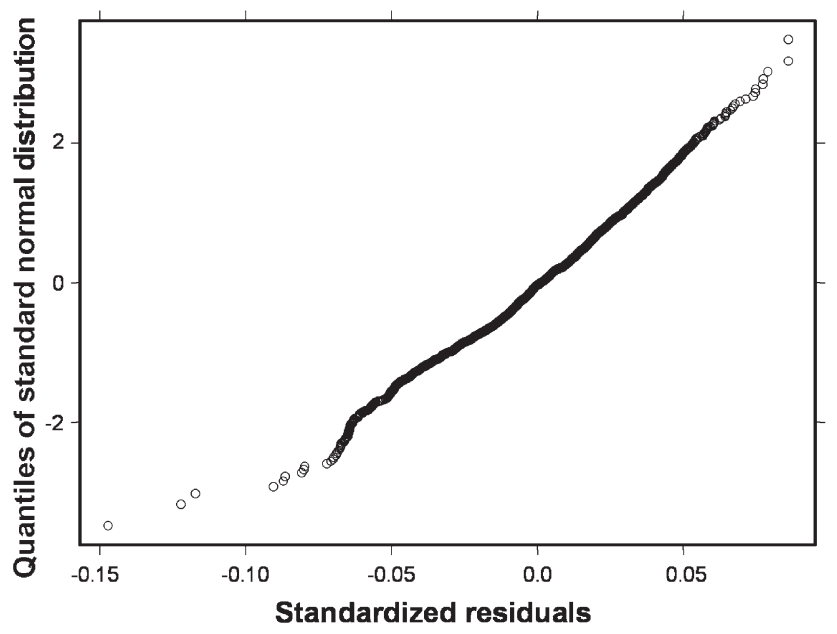

Fig. 5. Quantiles of standard normal distribution versus standardized residuals from final model used to evaluate sea lice burdens on farmed salmonids in southern Chile

improvement of fit obtained in our study for the random effects of geographical areas and zones within geographical areas suggests that the variability in sea lice infestations at these levels is mainly explained by the water temperature and salinity, species raised, weight of the fish, presence of current treatments and treatments 1 mo earlier and their interaction, pen shape, and mean parasite juvenile count at each level. Thus, after controlling for the other variables, the variability in the sea lice adult counts attributable to areas and zones within areas becomes negligible. Therefore, the parasite control measures at the areas and zone level should be focused on the factors described in the model that can be controlled by the manager, which are treatments, species farmed and pen shape. Of these options, the most feasible to be dynamically modified is treatment. The important association found between the reduction in the sea lice burden and the administration of 2 sequential treatments with avermectins (Table 5) suggests that this strategy is among the best options for achieving significant parasite reductions. Also, a producer considering installing a new facility in an area with historically high sea lice burdens, if feasible, should consider the culture of coho salmon rather than Atlantic salmon or rainbow trout.

\section{Variability between centers and within pens}

In contrast to the lack of significance associated with areas and zones, the variability between centers and within pens in a particular center was still important after controlling for the fixed variables. This means that there are 1 or more factors not included in this model that influence the differences in sea lice count at the center and pen level. The large variability found within pens may be explained by the presence of several factors that dynamically affect a fish population. For example, Johnson \& Albright (Johnson \& Albright 1992b) reported that under experimental conditions coho salmon implanted with cortisol are more susceptible to Lepeophtheirus salmonis, suggesting the importance of stress on sea lice burdens. MacKinnon (1998) described several stressors in fish potentially related to sea lice infestations. Hence, the large within-pen variability observed in the mean sea lice count may be explained by stressors varying in a pen over time, e.g. bacterial, viral and fungal infections, strong winds, transport, stock densities, handling and crowding.

Variability between centers is expected to be related to environmental and genetic factors because these are not uniform among centers. The transmission of juvenile parasites between centers over time might increase or decrease depending on changes in the local water currents (Costelloe et al. 1998) and possibly on the stochastic transmission of the parasite from native fish to farmed fish in sea pens. Differences in susceptibility to infestations with Caligus elongatus have been described for distinct full-sibling families of Atlantic salmon (Mustafa \& MacKinnon 1999), suggesting that variability between centers could also be due to genetic differences between fish populations at different centers. More research is needed to determine which breeds are more resistant to sea lice infestations in the field and also to describe and predict the pattern of transmission of sea lice between wild and farmed fish.

The significant interaction between date and pen effects suggests that the variability in parasite count at the pen level changes significantly as a function of date, and that this variability goes beyond the variables evaluated, including water temperature and salinity (effects that are traditionally related to date variations in sea lice count; Tully 1989). Thus, the date effect may be related to the previously described potential stressors, management factors, infections, etc. that may change over time.

Variability between pens

The very low variability between pens within a particular center was expected, considering that the managements of all the pens in a particular center are generally uniform and that pens within a center are usually closely related in space, allowing for spread of the sea lice infestations between pens. 
Spatial and temporal variability

A common observation in the salmon industry is that sea lice infestations are clustered in particular areas and zones (spatially), at specific times of the year (temporally) or even in particular areas at particular times (spatially and temporally). These observations suggest that there is correlation or dependency in sea lice counts between nearby centers because, if a neighboring center has a high sea lice burden and the environment conditions are favorable, there could be an effective spread of the parasite between the farms. The presence of such correlations could lead to under- or overestimation of the factors evaluated; therefore, future analyses should include these correlations by adjusting the models for the distance between centers. Such information could be included in a linear mixed model after obtaining the distance values from a geographical information system (GIS). These spatial and temporal dependencies are the subject of current research by the authors of this work.

\section{Fixed effects}

Treatments with oral emamectine benzoate have been reported to significantly reduce the adult parasite burden in Scottish farmed salmon 27 and $35 \mathrm{~d}$ after starting medication in autumn and winter, respectively (Stone et al. 2000). These findings are consistent with those of our study, in which 'current treatment' (treatment in the previous $15 \mathrm{~d}$ period) was not associated with a significantly lower mean parasite count, while treatment in the previous month was. Field trials in Chile using oral emamectine benzoate were effective even $95 \mathrm{~d}$ after treatment (Romero et al. 1999). Our results for treatments 2 and 3 mo earlier showed, when taking into account the other covariates in the model, no significant association with lower mean parasite numbers, suggesting that the effects of any treatment lasted no longer than $45 \mathrm{~d}$ (30 $\mathrm{d}$ for the significant treatment 1 mo earlier plus the $15 \mathrm{~d}$ sampling interval).

Interestingly, our results show no significant difference in the mean adult parasite count between pens treated 1 mo earlier with other treatments and pens not treated 1 mo earlier. This suggests that 'other treatments' included 'off-label' products (approved for other animal species but not for fish) that are not commonly reported in the program such as diflubenzuron, teflubenzuron and dichlorvos. These drugs have been described as being used in treating parasitized salmonids in Chile (Roth 2000) and are known only to act on the adult form of the parasite. Therefore, the lice burden may increase rapidly after the treatment if there are remaining juvenile-stage parasites following treatment at these locations.

Differences in fish-host susceptibility to Lepeophtheirus salmonis infestations have been well described (Nagasawa \& Takami 1993, Nagasawa et al. 1993, Mustafa \& MacKinnon 1999). Rainbow trout have been cited as the most susceptible species in Chile (Carvajal et al. 1998, Gonzalez et al. 2000). Our results support this hypothesis and, in addition, we describe a hierarchy in which rainbow trout is the most susceptible species, followed by Atlantic salmon and then coho salmon. The greater resistance of coho salmon to sea lice infestations may be related to the increased mucus production in this species. Parasite attachment to the fish skin may be impaired by both mechanical effects (sloughing) and the action of increased hydrolytic enzymes present in the mucus (Johnson \& Albright 1992a).

The significant association between host weight and mean parasite burden is most probably related to the time of exposure to the sea lice (age of the fish) and the larger surface exposed to the parasite in heavier fish, making these carry more lice in total but not necessarily per surface area. Recently, Tucker et al. (2002) described that under experimental conditions bigger fish had a greater total number of parasites attached than small individuals. However, when considering the densities of the parasites, small fish had a higher burden. Tucker et al. (2002) concluded that these differences are possible because smolts are most susceptible to sea lice infestations. Unfortunately, the authors did not provide compelling evidence to support the conclusion of greater susceptibility of the smolts to the infestations. In our study there were no significant quadratic or cubic associations, indicating that the relationship between fish weight and sea lice count was linear over the range of the weight measured.

The juvenile count was highly significant given that these forms mature to become adults in a short period of time (Johnson \& Albright 1991), reflecting the good prediction of adult counts that are obtained from the counts of juvenile forms.

The effect of water temperature on egg production and developmental stages of Lepeophtheirus salmonis and other caligid species has been extensively described under laboratory and field conditions (Hogans \& Trudeau 1989, Tully 1989, Johnson \& Albright 1991). The effect of salinity has been described in less detail, but it seems to mostly affect the time to maturation of the juvenile infective stage of the parasite (Johnson \& Albright 1991). Our results suggest that water temperature and salinity have a linear effect on sea lice adult counts, and the absence of statistically significant quadratic or cubic terms for these parameters may be due the small range of water temperatures and salinities included in the study, which approximately agrees 
with the described optimal temperature and salinity values for the development of the parasite $\left(10\right.$ to $15^{\circ} \mathrm{C}$ and 25 to $33 \mathrm{ppt}$ ).

The significant association between pen shape and sea lice burdens may be explained by several management factors not included in this study. For example, square pens have generally less volume than round pens and, thus, are typically stocked with fewer fish. Hence, if we assume that the difference in volume predisposes a smaller total population of lice in the square pens than in round pens, this might decrease the risk of individual fish being exposed to sea lice. As another example, if square pens are on average stocked less densely than round pens, this could decrease the stress on fish and also the fish exposure to sea lice populations $\left(\mathrm{m}^{-3}\right)$, resulting in a lower lice burden on fish in square pens. Unfortunately no data were available for densities or pen volume (only diameter) so these factors could not be accounted for in this study.

As no significant interaction term was found between pen 'shape' and the other covariates (e.g. 'species'), the pen effect is not expected to be an expression of another factor considered in this study that could be modifying the differences between the pen shapes.

We conclude that linear mixed-efffects modeling is an appropriate technique to identify the effects of different environmental and management factors on the variability in mean counts of adult sea lice infestations measured longitudinally on farmed salmonids in southern Chile. The results of this study suggest that the epidemiology of sea lice in farmed salmonids in southern Chile is complex, and additional studies are needed including the determination of factors that generate variability in the count of adult sea lice between and within pens and in the spatial and temporal patterns of the burdens.

Acknowledgements. The authors acknowledge Intesal SA for providing the database and the technical support. We also thank Messrs. J. L. Campisto and Crawford Revie and Drs. D. W. Hird, J. Cassigoli, L. Buttle, D. Nieto, S. Bartell, M. Thurmond, J. Case, D. Macias and G. Fosgate for their valuable comments and support during this project.

\section{LITERATURE CITED}

Boxaspen K (1997) Geographical and temporal variation in abundance of salmon lice (Lepeophtheirus salmonis) on salmon (Salmo salar L.). ICES J Mar Sci 54:1144-1147

Boxshall G, Bravo S (2000) On the identity of the common Caligus (Copepoda: Siphonostomatoida: Caligidae) from salmonid netpen systems in southern Chile. Contrib Zool 69:137-146

Carvajal J, Gonzalez L, George-Nascimento M (1998) Native sea lice (Copepoda: Caligidae) infestation of salmonids reared in netpen systems in southern Chile. Aquaculture 166:241-246
Costelloe M, Costelloe J, O'Donohoe G, Coghlan NJ, Oonk M, Van Der Heijden Y (1998) Planktonic distribution of sea lice larvae, Lepeophtheirus salmonis, in Killary Harbour, west coast of Ireland. J Mar Biol Assoc UK 78:853-874

Fernandez J, Villalba C (1986) Contribucion al conocimiento del genero caligus Muller, 1785 (Copepoda: Siphonostomatoida) en Chile. Gayana Zool 50:37-62

Gonzalez L, Carvajal J (1994) Parásitos en los cultivos marinos de salmónidos en el sur de Chile. Investig Pesq 38:87-96

Gonzalez L, Carvajal J, George-Nascimento M (2000) Differential infectivity of Caligus flexispina (Copepoda, Caligidae) in three farmed salmonids in Chile. Aquaculture 183: $13-23$

Hastein T, Bergsjo T (1976) The salmon lice Lepeophtheirus salmonis as the cause of disease in farmed salmonids. Riv Ital Piscic Ittiopatol A:3-4

Hogans WE (1995) Infection dynamics of sea lice, Lepeophtheirus salmonis (Copepoda: Caligidae): parasitic on Atlantic salmon (Salmo salar) cultured in marine waters of the Lower Bay of Fundy. Can Tech Rep Fish Aquat Sci 2067:101

Hogans WE, Trudeau DJ (1989) Preliminary studies on the biology of sea lice, Caligus elongatus, Caligus curtus and Lepeophtheirus salmonis (Copepoda: Caligidae) parasitic on cage-cultured salmonids in the lower Bay of Fundy [Canada]. Can Tech Rep Fish Aq Sci 1715:14 p

Jackson D, Deady S, Leahy Y, Hassett D (1997) Variations in parasitic caligid infestations on farmed salmonids and implications for their management. ICES J Mar Sci 54: 1104-1112

Jaworski A, Holm JC (1992) Distribution and structure of the population of sea lice, Lepeophtherius salmonis Kroyer, on Atlantic salmon, Salmo salar L., under typical rearing conditions. Aquacult Fish Manag 23:577-589

Johnson SC, Albright LJ (1991) Development, growth, and survival of Lepeophtheirus salmonis (Copepoda: Caligidae) under laboratory conditions. J Mar Biol Assoc UK 71:425-436

Johnson SC, Albright LJ (1992a) Comparative susceptibility and histopathology of the responses of naive Atlantic, chinook, and coho salmon to experimental infections with Lepeophterius salmonis (Copepoda: Caligidae). Dis Aquat Org 14:179-193

Johnson SC, Albright LJ (1992b) Effects of cortisol implants on the susceptibility and the histopathology of the responses of naive coho salmon Oncorhynchus kisutch to experimental infection with Lepeophtheirus salmonis (Copepoda: Caligidae). Dis Aquat Org 14:195-205

Kleinbaum DG (1994) Logistic regression: a self-learning text. Springer-Verlag, New York

Lindstrom M, Bates D (1990) Nonlinear mixed effects models for repeated measures Data. Biometrics 46:673-687

MacKinnon BM (1998) Host factors important in sea lice infections. ICES J Mar Sci 55:188-192

Mustafa A, MacKinnon BM (1999) Genetic variation in susceptibility of Atlantic salmon to the sea louse Caligus elongatus Nordmann, 1832. Can J Zool 77:1332-1335

Nagasawa K, Takami T (1993) Host utilization by the salmon louse Lepeophtheirus salmonis (Copepoda: Caligidae) in the Sea of Japan. J Parasitol 79:127-30

Nagasawa K, Ishida Y, Ogura M, Tadokoro K, Hiramatsu K (1993) The abundance and distribution of Lepeophtheirus salmonis (Copepoda: Caligidae) on six species of Pacific salmon in offshore waters of the North Pacific Ocean and Bering Sea. In: Boxshall G, Defaye D (eds) Pathogens of wild and farmed fish: sea lice. Ellis Horwood, Chichester, p 166-178

Pinheiro JC, Bates DM (2000) Mixed effects models in S and 
S-Plus. Springer-Verlag, New York

Revie CW, Gettinby G, Treasurer JW, Rae GH, Clark N, Mordue AJ, Pike AW (2002) Temporal, environmental and management factors influencing the epidemiological patterns of sea lice (Lepeophtheirus salmonis) infestations on farmed Atlantic salmon (Salmo salar) in Scotland. In: Salmon farming: towards an integrated pest management strategy for sea lice. Proceedings of a meeting. Aberdeen, UK, 18-19 June 2001. Pest Manag Sci 58:576-584

Reyes X, Bravo S (1983) Salmon coho Oncorhynchus kisutch, cultivado en Puerto Montt, Chile, nuevo huesped para el copepodo Caligus teres (Caligidae). Invest Mar Univ Catol Valpso 11:51-54

Romero M, Sutherland IH, Katz T, Endris RG (1999) Efficacy of SLICE ${ }^{\mathrm{TM}}$ against sea lice Caligus flexispina and Caligus teres on Atlantic salmon in Chile. In: Proceedings of the 4 th International Conference on Sea Lice, Dublin, Ireland, 28-30 June 1999. Aquacult Res 31

Roth M (2000) The availability and use of chemotherapeutic sea lice control products. Contrib Zool 69:109-118

Editorial responsibility: Chris Baldock, Brisbane, Australia
Stone J, Sutherland IH, Sommerville C, Richards RH, Varma KJ (2000) Commercial trials using emamectin benzoate to control sea lice Lepeophtheirus salmonis infestations in Atlantic salmon Salmo salar. Dis Aquat Org 41: 141-149

Tucker CS, Sommerville C, Wootten R (2002) Does size really matter? Effects of fish surface area on the settlement and initial survival of Lepeophtheirus salmonis, an ectoparasite of Atlantic salmon Salmo salar. Dis Aquat Org 49: $145-152$

Tully O (1989) The succession of generations and growth of the caligid copepods Caligus elongatus and Lepeophtheirus salmonis parasitizing farmed Atlantic salmon smolts (Salmo salar L.). J Mar Biol Assoc UK 69:279-288

Wilson K, Grenfell BT, Shaw DJ (1996) Analysis of aggregated parasite distributions: a comparison of methods. Funct Ecol 10:592-601

Wootten R (1985) Experience of sea lice infestations in Scottish salmon farms. Int Counc Explor Sea Comm Meet Maricult Comm F:7:4

Submitted: July 3, 2003; Accepted: July 9, 2004

Proofs received from author(s): April 6, 2005 